\title{
Riemann boundary-value problem for doubly-periodic bianalytic functions
}

\author{
Huili Han ${ }^{1 *}$, Hua Liu² and Yufeng Wang ${ }^{3}$
}

"Correspondence: nxhan@126.com 'School of Mathematics and Statistics, Ningxia University, Yinchuan, P.R. China

Full list of author information is available at the end of the article

\begin{abstract}
In this paper, we study Riemann boundary-value problem for doubly-periodic bianalytic functions. By the decomposition of doubly-periodic polyanalytic functions, the problem is transformed into two equivalent and independent Riemann boundary-value problems of doubly-periodic analytic functions, which has been discussed according to growth order of functions at the origin by Jianke Lu. Finally, we obtain the explicit expression of solutions and the conditions of solvability for the doubly-periodic bianalytic functions.
\end{abstract}

MSC: $30 \mathrm{G} 30 ; 45 \mathrm{E} 05$

Keywords: Doubly-periodic bianalytic function; Riemann boundary-value problem; Growth order; Canonical function; Freedom

\section{Introduction}

An extension of analytic function leads to polyanalytic function, which is usually defined as solutions of simple complex partial differential equation $\partial_{\bar{z}}^{n} f=0$, where $\partial_{\bar{z}}$ is the classical Cauchy-Riemann operator $\partial_{\bar{z}}=1 / 2[\partial / \partial x+i(\partial / \partial y)]$. Polyanalytic function stemmed from planar elasticity problems and was first investigated by Kolossov in 1908. A good overview of polyanalytic function is included in Balk's excellent monograph [1] or the literature [2]. Recently, various boundary-value problems (BVP) of polyanalytic functions and other functions determined by the general partial differential equations have been widely investigated by Begehr, Schmersau, Hile, Vanegas, Kumar, Jinyuan Du, Yufeng Wang, Ying Wang, Zhihua Du and others (see, for example, [1-21]). The general partial differential equations include the inhomogeneous polyanalytic equation [5], the higher order Poisson equation [6], and polyharmonic equations $[7,8]$.

Riemann BVP of single-periodic polyanalytic functions has been investigated [10, 11]. Analogously, a Riemann BVP of rotation-invariant polyanalytic functions has been discussed in [20]. Actually, a single-periodic polyanalytic function is defined by a translationinvariant group

$$
\mathcal{T}=\left\{\tau_{n}: \tau_{n}(z)=z+n \omega, n \in \mathbb{Z}\right\} \quad \text { with } \omega \in \mathbb{C} \backslash\{0\},
$$

which is generated by two elements $\left\{\tau_{1}, \tau_{-1}\right\}$ with $\tau_{ \pm 1}(z)=z \pm \omega$. Generally speaking, the single-periodic polyanalytic function is translation-invariant under the group $\mathcal{T}$, and the

(c) The Author(s) 2018. This article is distributed under the terms of the Creative Commons Attribution 4.0 International License (http://creativecommons.org/licenses/by/4.0/), which permits unrestricted use, distribution, and reproduction in any medium, provided you give appropriate credit to the original author(s) and the source, provide a link to the Creative Commons license, and indicate if changes were made. 
rotation-invariant polyanalytic function is invariant under a rotation group

$$
\mathcal{R}=\left\{\rho_{k}: \rho_{k}(z)=e^{\frac{2 k \pi i}{m}} z, k=0,1,2, \ldots, m-1\right\} .
$$

In general, single-periodic polyanalytic function and rotation-invariant polyanalytic function are automorphic.

In 1935, Natanzon first made use of doubly-periodic bianalytic function to deal with a problem on stresses deriving from a stretched plate. In 1957, Erwe also studied other classes of doubly-periodic polyanalytic functions. In 1982, Pokazeev further considered a general form of doubly-periodic polyanalytic functions. A concise history of investigation of doubly-periodic polyanalytic function has been introduced in the literature [2]. Doubly-periodic polyanalytic function is also automorphic and is determined by another translation-invariant group

$$
\mathcal{G}=\left\{\tau_{k, \ell}: \tau_{k, \ell}(z)=z+2 k \omega_{1}+2 \ell \omega_{2}, k, \ell \in \mathbb{Z}\right\} \quad \text { with } \operatorname{Im}\left(\omega_{2} / \omega_{1}\right) \neq 0
$$

Investigation of Riemann BVP for this kind of functions is a spontaneous thing.

For the compact Riemann surfaces of finite genus, the classical BVP of analytic functions was discussed in $[22,23]$. However, for the very important doubly-periodic problem, it is essential to have an effective method of solution which has been systematically investigated by Jianke $\mathrm{Lu}$ [24]. Later, BVP of automorphic analytic functions has been first discussed by Gakhov and Chibrikova [25, 26].

Up to now, Riemann BVP for doubly-periodic polyanalytic function has not been wellposed and systematically investigated. In this article, our main objective is to set up the theory of doubly-periodic bianalytic function. The way to solve this problem is the conversion method used in [16]. Riemann BVP for doubly-periodic polyanalytic functions will be presented in the forthcoming paper.

This article is organized as follows. In Sect. 2, we give a decomposition of doublyperiodic polyanalytic functions, which will be used to solve BVPs of doubly-periodic bianalytic functions. It is worth mentioning that the decomposition obtained here is distinct from the classical decomposition described in [2]. In Sect. 3, the growth order of doubly-periodic polyanalytic functions at the origin is defined. To pose the reasonable BVPs of doubly-periodic bianalytic functions, the definition of growth order at the origin is needed. In the classical monographs [24, 25], the growth order of doubly-periodic functions is not explicitly defined. In Sect. 4, Riemann BVP of doubly-periodic bianalytic functions is presented. The solutions and conditions of solvability of this kind of problem are obtained by Jianke Lu [24]. By the decomposition of doubly-periodic bianalytic functions, the problem is transformed into two independent Riemann-type BVPs of doubly-periodic analytic functions. Finally, the solution is explicitly expressed as an integral representation.

\section{Doubly-periodic polyanalytic functions}

Without loss of generality, we always assume that $\operatorname{Im}\left(\omega_{2} / \omega_{1}\right)>0$ in the following. The parallelogram with vertices $\omega_{1}+\omega_{2},-\omega_{1}+\omega_{2},-\omega_{1}-\omega_{2}$, and $\omega_{1}-\omega_{2}$ is denoted by $S_{0}$, which is usually called the fundamental cell. Obviously, the origin is the center of the fundamental cell $S_{0}$. 
The classical Weierstrass's $\zeta$-function is defined by

$$
\zeta(z)=\frac{1}{z}+\sum_{(k, \ell) \neq(0,0)}\left(\frac{1}{z-\Omega_{k \ell}}+\frac{1}{\Omega_{k \ell}}+\frac{z}{\Omega_{k \ell}^{2}}\right),
$$

with $\Omega_{k \ell}=2 k \omega_{1}+2 \ell \omega_{2}$, and $k, l$ are integers. Clearly,

$$
\zeta\left(z+2 \omega_{j}\right)=\zeta(z)+2 \eta_{j}, \quad j=1,2,
$$

where $\eta_{j}=\zeta\left(\omega_{j}\right)$ satisfies the relation

$$
2 \eta_{1} \omega_{2}-2 \eta_{2} \omega_{1}=\pi i
$$

Let

$$
\phi(z)=\bar{z}-\lambda z-\delta \zeta(z)
$$

with

$$
\lambda=\frac{2 i}{\pi}\left(\bar{w}_{1} \eta_{2}-\bar{w}_{2} \eta_{1}\right), \quad \delta=-\frac{2 i}{\pi}\left(\bar{w}_{1} w_{2}-\bar{w}_{2} w_{1}\right)
$$

By simple computation, one has

$$
\phi\left(z+2 \omega_{j}\right)=\overline{z+2 \omega_{j}}-\lambda\left(z+2 \omega_{j}\right)-\delta \zeta\left(z+2 \omega_{j}\right)=\phi(z), \quad z \neq 0 .
$$

This implies that $\phi$ is a doubly-periodic bianalytic function.

If the open set $\Omega$ on the complex plane $\mathbb{C}$ satisfies the condition $z+2 k \omega_{1}+2 \ell \omega_{2} \in \Omega$ for $\forall z \in \Omega, \forall k, \ell \in \mathbb{Z}$, then $\Omega$ is called a doubly-periodic open set with periods $2 \omega_{1}, 2 \omega_{2}$. Similar to the definition of single-periodic polyanalytic function in [10], we give the following definition.

Definition 2.1 Suppose $f$ to be a polyanalytic function [1] of order $n$ on $\Omega$, where $\Omega$ is a doubly-periodic open set with periods $2 \omega_{1}, 2 \omega_{2}$. If

$$
f\left(z+2 \omega_{j}\right)=f(z), \quad \forall z \in \Omega, j=1,2,
$$

then we say that $f$ is a doubly-periodic polyanalytic function of order $n$ with periods $2 \omega_{1}, 2 \omega_{2}$ on $\Omega$, or simply doubly-periodic polyanalytic function. The collection of all the doubly-periodic polyanalytic functions on $\Omega$ is denoted by $D P H_{n}(\Omega)$.

By Definition 2.1, $D P H_{1}(\Omega)$ is just a set of all the doubly-periodic analytic functions on the doubly-periodic open set $\Omega$. The function $f \in D P H_{1}(\Omega)$ is called doubly-periodic bianalytic function. $D P H_{n}(\Omega)$ is a subset of the collection of polyanalytic functions on $\Omega$ denoted by $H_{n}(\Omega)=\left\{f: \partial_{\bar{z}}^{n} f(z)=0, z \in \Omega\right\}$. Equation (2.5) is equivalent to

$$
\partial_{\bar{z}}^{k} f\left(z+2 \omega_{j}\right)=\partial_{\bar{z}}^{k} f(z), \quad \forall z \in \Omega \text { for } k=0,1, \ldots, n-1 \text { and } j=1,2
$$


Now we introduce the subset of $D P H_{n}(\Omega)$ as follows:

$$
D P H_{n}\left(\Omega ; 2 \omega_{1}, 2 \omega_{2}\right)=\left\{f \in D P H_{n}(\Omega): f\left(z+2 \omega_{j}\right)=f(z), \forall z \in \Omega \text { for } j=1,2\right\},
$$

which is an object of investigation in the following.

Finally, one arrives at the decomposition of doubly-periodic polyanalytic functions, used to solve Riemann BVP of doubly-periodic bianalytic functions in the sequel.

Theorem 2.1 Let $\Omega$ be a doubly-periodic open set with periods $2 \omega_{1}, 2 \omega_{2}$ and $0 \notin \Omega$. Then

$$
\begin{aligned}
D P H_{n}\left(\Omega ; 2 \omega_{1}, 2 \omega_{2}\right)= & D P H_{1}\left(\Omega ; 2 \omega_{1}, 2 \omega_{2}\right) \\
& \oplus[\bar{z}-\lambda z-\delta \zeta(z)] D P H_{1}\left(\Omega ; 2 \omega_{1}, 2 \omega_{2}\right) \\
& \oplus \cdots \oplus[\bar{z}-\lambda z-\delta \zeta(z)]^{n-1} D P H_{1}\left(\Omega ; 2 \omega_{1}, 2 \omega_{2}\right),
\end{aligned}
$$

with $[\bar{z}-\lambda z-\delta \zeta(z)]^{j} D P H_{1}\left(\Omega ; 2 \omega_{1}, 2 \omega_{2}\right)=\left\{[\bar{z}-\lambda z-\delta \zeta(z)]^{j} f(z): f \in D P H_{1}\left(\Omega ; 2 \omega_{1}, 2 \omega_{2}\right)\right\}$ for $j=0,1, \ldots, n-1$, where $\zeta$ is defined by (2.1), and $\lambda$, $\delta$ are given by (2.4).

Proof We only need to verify the relation $\subseteq$ by induction. Obviously, if $n=1$, the theorem is straightforward. Suppose that the relation

$$
\begin{aligned}
D P H_{n-1}\left(\Omega ; 2 \omega_{1}, 2 \omega_{2}\right) \subseteq & D P H_{1}\left(\Omega ; 2 \omega_{1}, 2 \omega_{2}\right) \\
& \oplus[\bar{z}-\lambda z-\delta \zeta(z)] D P H_{1}\left(\Omega ; 2 \omega_{1}, 2 \omega_{2}\right) \\
& \oplus \cdots \oplus[\bar{z}-\lambda z-\delta \zeta(z)]^{n-2} D P H_{1}\left(\Omega ; 2 \omega_{1}, 2 \omega_{2}\right)
\end{aligned}
$$

is valid. Next one has to verify

$$
\begin{aligned}
D P H_{n}\left(\Omega ; 2 \omega_{1}, 2 \omega_{2}\right) \subseteq & D P H_{1}\left(\Omega ; 2 \omega_{1}, 2 \omega_{2}\right) \\
& \oplus[\bar{z}-\lambda z-\delta \zeta(z)] D P H_{1}\left(\Omega ; 2 \omega_{1}, 2 \omega_{2}\right) \\
& \oplus \cdots \oplus[\bar{z}-\lambda z-\delta \zeta(z)]^{n-1} D P H_{1}\left(\Omega ; 2 \omega_{1}, 2 \omega_{2}\right) .
\end{aligned}
$$

Let $f \in D P H_{n}\left(\Omega ; 2 \omega_{1}, 2 \omega_{2}\right)$. Then $\partial_{\bar{z}} f \in D P H_{n-1}\left(\Omega ; 2 \omega_{1}, 2 \omega_{2}\right)$. And hence, by the inductive hypothesis, there exist $g_{j}(z) \in D P H_{1}\left(\Omega ; 2 \omega_{1}, 2 \omega_{2}\right), j=0,1, \ldots, n-2$, such that

$$
\partial_{\bar{z}} f(z)=g_{0}(z)+\sum_{j=1}^{n-2} \frac{[\bar{z}-\lambda z-\delta \zeta(z)]^{j}}{j !} g_{j}(z), \quad z \in \Omega .
$$

Setting

$$
h(z)=f(z)-[\bar{z}-\lambda z-\delta \zeta(z)] g_{0}(z)-\sum_{j=1}^{n-2} \frac{[\bar{z}-\lambda z-\delta \zeta(z)]^{j+1}}{(j+1) !} g_{j}(z), \quad z \in \Omega,
$$

one has, by (2.9),

$$
\partial_{\bar{z}} h(z)=\partial_{\bar{z}} f(z)-g_{0}(z)-\sum_{j=1}^{n-2} \frac{[\bar{z}-\lambda z-\delta \zeta(z)]^{j}}{j !} g_{j}(z)=0, \quad z \in \Omega .
$$


Therefore, $h \in D P H_{1}\left(\Omega ; 2 \omega_{1}, 2 \omega_{2}\right)$. And (2.10) is rewritten as

$$
f(z)=h(z)+[\bar{z}-\lambda z-\delta \zeta(z)] g_{0}(z)+\sum_{j=1}^{n-2} \frac{[\bar{z}-\lambda z-\delta \zeta(z)]^{j+1}}{(j+1) !} g_{j}(z), \quad z \in \Omega
$$

which implies that $(2.8)$ remains true.

Theorem 2.1 indicates that $f \in D P H_{n}\left(\Omega ; 2 \omega_{1}, 2 \omega_{2}\right)$ admits a unique decomposition

$$
f(z)=\sum_{j=0}^{n-1}[\bar{z}-\lambda z-\delta \zeta(z)]^{j} f_{j}(z), \quad z \in \Omega
$$

where $f_{j} \in D P H_{1}\left(\Omega ; 2 \omega_{1}, 2 \omega_{2}\right)$ is called $j$-component of $f$ with respect to the base $\{[\bar{z}-\lambda z-$ $\left.\delta \zeta(z)]^{j}: j=0,1,2, \ldots, n-1\right\}$. Specially, $g \in D P H_{2}\left(\Omega ; 2 \omega_{1}, 2 \omega_{2}\right)$ has the unique expansion

$$
g(z)=g_{1}(z)+[\bar{z}-\lambda z-\delta \zeta(z)] g_{2}(z), \quad z \in \Omega
$$

with $g_{j} \in D P H_{1}\left(\Omega ; 2 \omega_{1}, 2 \omega_{2}\right), j=1,2$.

\section{Growth order of doubly-periodic bianalytic functions}

First, the following definition is analogous to that in [10]. This is just the definition of growth order for the general polyanalytic function at the origin [15].

Definition 3.1 Suppose $\Omega$ to be a doubly-periodic open set with periods $2 \omega_{1}, 2 \omega_{2}, 0 \in \Omega$ and $f \in D P H_{n}\left(\Omega ; 2 \omega_{1}, 2 \omega_{2}\right)$. If there exists an integer $m$ such that

$$
\limsup _{z \in \Omega, z \rightarrow 0}\left|z^{m} f(z)\right|=\alpha, \quad \alpha \in(0,+\infty)
$$

then we say that $f$ possesses order $m$ at the origin, denoted by $\operatorname{Ord}(f, 0)=m$. If

$$
\limsup _{z \in \Omega, z \rightarrow 0}\left|z^{m} f(z)\right|=+\infty \quad \text { for any } m \in \mathbb{Z}
$$

then we say that $f$ has order $+\infty$ at the origin, denoted by $\operatorname{Ord}(f, 0)=+\infty$. We assume $\operatorname{Ord}(f, 0)=-\infty$ if and only if $f=0$.

Now, one has the following result needed in the sequel.

Lemma 3.1 Suppose $\Omega$ to be a doubly-periodic open set with periods $2 \omega_{1}, 2 \omega_{2}, 0 \in \Omega$ and $f \in D P H_{1}\left(\Omega ; 2 \omega_{1}, 2 \omega_{2}\right)$. At the deleted neighbor of the origin, one has

$$
f(z)=c_{0}(z)+\sum_{j=1}^{\infty} c_{j} \zeta^{(j)}(z)
$$

where $\zeta$ is defined by (2.1), the order of $c_{0}(z) \in D P H_{1}\left(\Omega ; 2 \omega_{1}, 2 \omega_{2}\right)$ is not more than 1 and $c_{j} \in \mathbb{C}, j=1,2, \ldots$. 
Proof Since $0 \in \Omega$ and $f \in D P H_{1}\left(\Omega ; 2 \omega_{1}, 2 \omega_{2}\right)$, at the deleted neighbor of the origin, one has Laurent's expansion

$$
f(z)=\sum_{j=-\infty}^{+\infty} d_{j} \frac{1}{z^{j}}, \quad d_{j}=\frac{1}{2 \pi i} \int_{|t|=r} f(t) t^{j-1} \mathrm{~d} t
$$

for sufficiently small $r>0$. Let

$$
\zeta(z)=\frac{1}{z}+\zeta_{0}(z), \quad \zeta_{0}(z)=\sum_{(k, \ell) \neq(0,0)}\left(\frac{1}{z-\Omega_{k \ell}}+\frac{1}{\Omega_{k \ell}}+\frac{z}{\Omega_{k \ell}^{2}}\right),
$$

where $\zeta_{0}(0)=0$ and $\zeta_{0}(z)$ is analytic at the origin. Thus one gets

$$
\zeta^{(j)}(z)=\frac{(-1)^{j}}{z^{j+1}}+\zeta_{0}^{(j)}(z), \quad j \in \mathbb{Z}^{+}
$$

which is equivalent to

$$
\frac{1}{z^{j}}=(-1)^{j-1}\left[\zeta^{(j-1)}(z)-\zeta_{0}^{(j-1)}(z)\right], \quad j \in \mathbb{Z}^{+} .
$$

Inserting (3.4) into (3.2), we get

$$
f(z)=d(z)+\sum_{j=1}^{\infty} d_{j+1}(-1)^{j} \zeta^{(j)}(z)
$$

with

$$
d(z)=\sum_{j=-1}^{+\infty} d_{-j} z^{j}-\sum_{j=1}^{\infty} d_{j+1}(-1)^{j} \zeta_{0}^{(j)}(z) \in D P H_{1}\left(\Omega ; 2 \omega_{1}, 2 \omega_{2}\right) .
$$

The order of $d(z)$ is obviously not more than 1 . This completes the proof.

Corollary 3.1 Suppose that $f \in D P H_{1}\left(\mathbb{C} ; 2 \omega_{1}, 2 \omega_{2}\right)$ possesses a uniquely possible singular point $z=0$ in the fundamental cell $S_{0}$. Then one has

$$
f(z)=c_{0}+\sum_{j=1}^{\infty} c_{j} \zeta^{(j)}(z), \quad z \in S_{0} \backslash\{0\}
$$

where $\zeta$ is defined by (2.1), and $c_{j} \in \mathbb{C}, j=0,1,2, \ldots$.

Proof By Lemma 3.1, at the deleted neighbor of the origin, one has

$$
f(z)=c_{0}(z)+\sum_{j=1}^{\infty} c_{j} \zeta^{(j)}(z)
$$

where $\zeta$ is defined by (2.1), the order of $c_{0}(z) \in D P H_{1}\left(\Omega ; 2 \omega_{1}, 2 \omega_{2}\right)$ is not more than 1 and $c_{j} \in \mathbb{C}, j=1,2, \ldots$. And $c_{0}(z) \in D P H_{1}\left(\mathbb{C} ; 2 \omega_{1}, 2 \omega_{2}\right)$ implies that $c_{0}(z)$ is an elliptic function. By Liouville's theorem, $c_{0}(z)$ is a constant function. 
Lemma 3.2 Suppose $\Omega$ to be a doubly-periodic open set with periods $2 \omega_{1}, 2 \omega_{2}, 0 \in \Omega$ and $f \in D_{P P H}\left(\Omega ; 2 \omega_{1}, 2 \omega_{2}\right)$. If $\operatorname{Ord}(f, 0) \leq m$, then $\operatorname{Ord}\left(f_{1}, 0\right) \leq m+2$ and $\operatorname{Ord}\left(f_{2}, 0\right) \leq m+1$, where $f_{j}$ is j-component off.

Proof First, this theorem is verified under $m>1$. By Theorem 2.1, there exist $f_{1}, f_{2} \in$ $D P H_{1}\left(\Omega ; 2 \omega_{1}, 2 \omega_{2}\right)$ such that $f(z)=f_{1}(z)+\phi(z) f_{2}(z)$. This leads to

$$
\begin{aligned}
f(z) & =\left\{f_{1}(z)-[\lambda z+\delta \zeta(z)] f_{2}(z)\right\}+\bar{z} f_{2}(z) \\
& =\sum_{j=-\infty}^{\infty} a_{j} z^{j}+\bar{z} \sum_{k=-\infty}^{\infty} b_{k} z^{k}
\end{aligned}
$$

near the origin, where $a_{j}, b_{k}$ are constants.

Let $\ell \in \mathbb{Z}^{+}$and $\ell \geq m$. We choose sufficiently small $r>0$ such that $D_{r}=\{z:|z|<r\} \subseteq \Omega$. By (3.6), one has

$$
\begin{aligned}
\frac{1}{2 \pi i} \int_{|t|=r} f(t) t^{\ell} \mathrm{d} t & =\frac{1}{2 \pi i} \int_{|t|=r}\left(\sum_{j=-\infty}^{\infty} a_{j} t^{j}+\frac{r^{2}}{t} \sum_{k=-\infty}^{\infty} b_{k} t^{k}\right) t^{\ell} \mathrm{d} t \\
& =\frac{1}{2 \pi i} \int_{|t|=r} \frac{a_{-(\ell+1)}}{t} \mathrm{~d} t+r^{2} \frac{1}{2 \pi i} \int_{|t|=r} \frac{b_{-\ell}}{t} \mathrm{~d} t \\
& =a_{-(\ell+1)}+r^{2} b_{-\ell} .
\end{aligned}
$$

Now $\operatorname{Ord}(f, 0) \leq m$ implies that there exist $M>0, r_{1}>0$ such that

$$
|f(z)| \leq \frac{M}{|z|^{m}}, \quad|z|<r_{1} .
$$

We assume $0<r<r_{1}$, and one has the estimation

$$
\left|\frac{1}{2 \pi i} \int_{|t|=r} f(t) t^{\ell} \mathrm{d} t\right| \leq M r^{\ell+1-m} .
$$

Combining (3.7) with (3.8), we get

$$
\left|a_{-(\ell+1)}\right|+r^{2}\left|b_{-\ell}\right| \leq M r^{\ell+1-m}
$$

for sufficiently small $r>0$. Let $r \rightarrow 0^{+}$, and one has

$$
a_{-(\ell+1)}=0, \quad \ell \geq m .
$$

This leads to

$$
\operatorname{Ord}\left(f_{1}-[\lambda z+\delta \zeta(z)] f_{2}, 0\right) \leq m
$$

Therefore,

$$
\operatorname{Ord}\left(f_{2}, 0\right)=\operatorname{Ord}\left(\bar{z} f_{2}, 0\right)+1 \leq \max \left\{\operatorname{Ord}(f, 0), \operatorname{Ord}\left(f_{1}-[\lambda z+\delta \zeta(z)] f_{2}, 0\right)\right\}+1 \leq m+1
$$


and

$\operatorname{Ord}\left(f_{1}, 0\right) \leq \max \left\{\operatorname{Ord}(f, 0), \operatorname{Ord}\left(\phi f_{2}, 0\right)\right\} \leq m+2$,

where $\phi$ is defined by (2.3).

Finally, if $m \leq 1$, by Corollary 3.1 in [15], similar to the discussion above, it is not difficult to know that the conclusion remains true.

In what follows, we need the operators

$$
\mathcal{L}_{j}[f]=d_{j}, \quad j \in \mathbb{Z},
$$

where $d_{j}$ is the $j$-coefficient of Laurent's expansion of the function $f$ defined in (3.2).

Theorem 3.1 Suppose $\Omega$ to be a doubly-periodic open set with periods $2 \omega_{1}, 2 \omega_{2}, 0 \in \Omega$ and $f \in D P H_{2}\left(\Omega ; 2 \omega_{1}, 2 \omega_{2}\right)$. Then $\operatorname{Ord}(f, 0) \leq m$ if and only if

$$
\operatorname{Ord}\left(f_{j}, 0\right) \leq m+3-j, \quad j=1,2,
$$

and

$$
\mathcal{L}_{j+1}\left[f_{1}\right]=\delta \mathcal{L}_{j}\left[f_{2}\right], \quad j=m, m+1,
$$

where $\mathcal{L}_{j}$ is the operator defined by (3.9), and $\delta$ is given in (2.4).

Proof First, we assume $m>3$. And we prove the necessity. By Theorem 2.1, there exist $f_{1}, f_{2} \in D P H_{1}\left(\Omega ; 2 \omega_{1}, 2 \omega_{2}\right)$ such that $f(z)=f_{1}(z)+\phi(z) f_{2}(z)$. By Lemma 3.2, $\operatorname{Ord}(f, 0) \leq m$ implies $\operatorname{Ord}\left(f_{1}, 0\right) \leq m+2$ and $\operatorname{Ord}\left(f_{2}, 0\right) \leq m+1$. Also by Lemma 3.1,

$$
\begin{aligned}
& f_{1}(z)=c_{0}(z)+\sum_{j=1}^{m+1} c_{j} \zeta^{(j)}(z), \\
& f_{2}(z)=d_{0}(z)+\sum_{j=1}^{m} d_{j} \zeta^{(j)}(z) .
\end{aligned}
$$

Inserting (3.12) and (3.13) into the expression $f(z)=f_{1}(z)+\phi(z) f_{2}(z)$, one easily gets

$$
\begin{aligned}
f(z)= & c_{0}(z)+\sum_{k=1}^{m+1} c_{k} \zeta^{(k)}(z) \\
& +[\bar{z}-\lambda z-\delta \zeta(z)]\left[d_{0}(z)+\sum_{j=1}^{m} d_{j} \zeta^{(j)}(z)\right]
\end{aligned}
$$

Thus, $\operatorname{Ord}(f, 0) \leq m$, where $f$ given in (3.14), if and only if

$$
P . P\left(c_{k+1} \zeta^{(k+1)}(z)-\delta d_{k} \zeta(z) \zeta^{(k)}(z), 0\right)=0, \quad k=m-1, m,
$$


where P.P $(h, 0)$ denotes the principal part of $h$. Equation (3.15) is equivalent to

$$
d_{k}=\frac{c_{k+1}}{\delta}, \quad k=m-1, m
$$

Inserting this expression into (3.14), one has

$$
\begin{aligned}
f(z)= & c_{0}(z)+\sum_{k=1}^{m-1} c_{k} \zeta^{(k)}(z)+c_{m} \zeta^{(m)}(z)+c_{m+1} \zeta^{(m+1)}(z) \\
& +[\bar{z}-\lambda z-\delta \zeta(z)]\left[d_{0}(z)+\sum_{j=1}^{m-2} d_{j} \zeta^{(j)}(z)+\frac{c_{m}}{\delta} \zeta^{(m-1)}(z)+\frac{c_{m+1}}{\delta} \zeta^{(m)}(z)\right],
\end{aligned}
$$

where $c_{0}(z), d_{0}(z) \in D P H_{1}\left(\Omega ; 2 \omega_{1}, 2 \omega_{2}\right), \operatorname{Ord}\left(c_{0}, 0\right) \leq 1, \operatorname{Ord}\left(d_{0}, 0\right) \leq 1$, and $d_{j}, c_{k} \in \mathbb{C}$. Therefore, (3.11) is true.

The sufficiency is obvious. Finally, if $m \leq 3$, analogously to the discussion above, the conclusion is also true. This completes the proof of this theorem.

\section{Riemann BVP for doubly-periodic analytic functions}

In this section, we will give the solutions and conditions of solvability for Riemann BVP for doubly-periodic analytic functions, which was investigated in detail by Jianke $\mathrm{Lu}$ [24].

Let $L_{0}$ be a closed smooth Jordan curve, oriented counterclockwise. The fundamental cell $S_{0}$ is divided into two domains denoted by $S_{0}^{+}$and $S_{0}^{-}$, respectively. Without loss of generality, we always assume $0 \in S_{0}^{+}$. Let $L_{k, \ell}=2 k \omega_{1}+2 \ell \omega_{2}+L_{0}$ for $k, \ell \in \mathbb{Z}, S^{+}=$ $\bigcup_{k, \ell \in \mathbb{Z}}\left(2 k \omega_{1}+2 \ell \omega_{2}+S_{0}^{+}\right), S^{-}=\mathbb{C} \backslash \overline{S^{+}}$, and we assume that $L_{k, \ell}$ has the same orientation as $L_{0}$ for every $k, \ell \in \mathbb{Z}$. For the convenience, we set $L=\bigcup_{k, \ell \in \mathbb{Z}} L_{k, \ell}$.

Now, our problem is to find a sectionally doubly-periodic analytic function $\Phi(z) \in$ $D P H_{1}\left(\Omega ; 2 \omega_{1}, 2 \omega_{2}\right)$ satisfying a boundary condition and a growth condition

$$
\left\{\begin{array}{l}
\Phi^{+}(t)=G(t) \Phi^{-}(t)+g(t), \quad t \in L \\
\operatorname{Ord}(\Phi, 0) \leq m
\end{array}\right.
$$

where the given Hölder-continuous functions $G, g$ satisfy $G\left(t+2 \omega_{j}\right)=G(t), g\left(t+2 \omega_{j}\right)=g(t)$, $j=1,2$ and $G(t) \neq 0, t \in L$. This problem is simply called $D R_{m}$ problem.

Introduce the function

$$
\mu(z)=\frac{\sigma(z) \sigma\left(z-\omega_{1}-\omega_{2}\right)}{\sigma\left(z-\omega_{1}\right) \sigma\left(z-\omega_{2}\right)},
$$

with

$$
\sigma(z)=z \prod_{(k, \ell) \neq(0,0)}\left(1-\frac{z}{2 k \omega_{1}+2 \ell \omega_{2}}\right) \exp \left(\frac{z}{2 k \omega_{1}+2 \ell \omega_{2}}+\frac{z^{2}}{2 k \omega_{1}+2 \ell \omega_{2}}\right) .
$$

Then $\mu$ is an elliptic function with periods $2 \omega_{1}, 2 \omega_{2}$ and $\operatorname{Ord}(\mu, 0)=-1$. Let

$$
\kappa=\frac{1}{2 \pi}[G(t)]_{L_{0}}
$$


which is called the index, and

$$
G_{*}=\frac{1}{2 \pi i} \int_{L_{0}} \log \left[\mu^{-\kappa}(t) G(t)\right] \mathrm{d} t .
$$

Without loss of generality, we assume $G_{*} \notin L$ in the following. The solutions and conditions of solvability of $D R_{m}$ problem (4.1) are presented in two cases.

\subsection{The case $G_{*}=2 k \omega_{1}+2 \ell \omega_{2}$ for some $k, \ell \in \mathbb{Z}$}

In this case, $G_{*}=0\left(\bmod 2 \omega_{1}, 2 \omega_{2}\right)$. Let

$$
X(z)= \begin{cases}\mu^{\kappa}(z) h(z) e^{\Gamma(z)}, & z \in S^{+}, \\ h(z) e^{\Gamma(z)}, & z \in S^{-}\end{cases}
$$

where

$$
\Gamma(z)=\frac{1}{2 \pi i} \int_{L_{0}} \log \left[\mu^{-\kappa}(t) G(t)\right] \zeta(t-z) \mathrm{d} t, \quad z \in S^{+} \cup S^{-}
$$

and

$$
h(z)=\exp \left[2\left(k \eta_{1}+\ell \eta_{2}\right) z\right]
$$

In (4.8), $\eta_{j}=\zeta\left(\omega_{j}\right), j=1,2 . X$ defined by (4.6) is called the canonical function which possesses the following five properties:

(1) $X \in D P H_{1}\left(\Omega ; 2 \omega_{1}, 2 \omega_{2}\right)$;

(2) $X^{+}(t)=G(t) X^{-}(t), t \in L$;

(3) $X^{ \pm}(t) \in H(L)$;

(4) $X(z) \neq 0, z \neq 2\left(p \omega_{1}+q \omega_{2}\right)$ with $p, q \in \mathbb{Z}$ and $X^{ \pm}(t) \neq 0$ for $t \in L$;

(5) $X(z)$ has a pole of order $-\kappa$ at the origin, or say $\operatorname{Ord}(X, 0)=-\kappa$.

If the function $Y$ also satisfies five properties from (1) to (5) above, then there exists $C \in \mathbb{C}$ such that $Y(z)=C X(z)$, where $X$ is given by (4.6).

For convenience, we introduce the set of elliptic functions of order $k$ with an exclusive singular point $z=0$ as follows:

$$
\Pi_{k}(\zeta)= \begin{cases}\left\{c_{0}+c_{1} \zeta^{\prime}(z)+\cdots+c_{k} \zeta^{(k)}(z): c_{j} \in \mathbb{C}, j=0,1, \ldots, k\right\}, & k>0, \\ \mathbb{C}, & k=0, \\ \{0\}, & k<0 .\end{cases}
$$

Now we state the results obtained by Jianke Lu.

Theorem 4.1 Under this case, two subcases arise:

(1) When $\kappa+m>0, D R_{m}$ problem (4.1) is solvable and its solution can be expressed as

$$
\begin{aligned}
\Phi(z)= & \frac{X(z)}{2 \pi i} \int_{L_{0}} \frac{g(t)}{X^{+}(t)}[\zeta(t-z)+\zeta(z)] \mathrm{d} t \\
& +X(z) p_{\kappa+m-1}(z), \quad p_{\kappa+m-1} \in \Pi_{\kappa+m-1}(\zeta),
\end{aligned}
$$

where $\Pi_{\kappa+m-1}(\zeta)$ is defined by (4.9) and $X$ is given by (4.6). 
(2) When $\kappa+m \leq 0$, if and only if

$$
\frac{1}{2 \pi i} \int_{L_{0}} \frac{g(t)}{X^{+}(t)} \zeta^{(k)}(t) \mathrm{d} t=0, \quad k=-1,0,1,2, \ldots,-\kappa-m-1,
$$

$D R_{m}$ problem (4.1) is solvable and its solution can be written as

$$
\Phi(z)=\frac{X(z)}{2 \pi i} \int_{L_{0}} \frac{g(t)}{X^{+}(t)}[\zeta(t-z)-\zeta(t)] \mathrm{d} t+C X(z), \quad C \in \Pi_{\kappa+m}(\zeta) .
$$

We assume $\zeta^{(-1)}(t)=\zeta^{(0)}(t)=1$ in (4.11).

In general, the freedom of solutions is $\kappa+m$.

\subsection{The case $G_{*} \neq 2 k \omega_{1}+2 \ell \omega_{2}$ for any $k, \ell \in \mathbb{Z}$}

In this case, there exists $G_{0} \in S_{0}$ such that $G_{*}=G_{0}\left(\bmod 2 \omega_{1}, 2 \omega_{2}\right)$, and $G_{0} \neq 0$. Let $X$ be defined by (4.6), where

$$
h(z)=\frac{\sigma(z)}{\sigma\left(z-G_{*}\right)} .
$$

At this time, $X$ is also the canonical function which satisfies four properties from (1) to (4) in Sect. 4.1, and

(5) $X(z)$ has a pole of order $-\kappa-1$ at the origin, precisely $\operatorname{Ord}(X, 0)=-\kappa-1$.

The following result is also obtained by Jianke Lu. $X$ used in the following theorem is defined by (4.6) with (4.13).

Theorem 4.2 Under this case, two subcases arise:

(1) When $\kappa+m+1 \geq 0$, if and only if the condition of solvability

$$
\frac{1}{2 \pi i} \int_{L_{0}} \frac{g(t)}{X^{+}(t)} \zeta^{(k)}(t) \mathrm{d} t=0, \quad k=0,1,2, \ldots,-\kappa-m-1
$$

is fulfilled, $D R_{m}$ problem (4.1) is solvable and its solution can be expressed as

$$
\begin{aligned}
\Phi(z)= & \frac{X(z)}{2 \pi i} \int_{L_{0}} \frac{g(t)}{X^{+}(t)}\left[\zeta(t-z)+\zeta(z)-\zeta\left(t-G_{0}\right)-\zeta\left(G_{0}\right)\right] \mathrm{d} t \\
& +X(z)\left[p_{\kappa+m}(z)-p_{\kappa+m}\left(G_{0}\right)\right]
\end{aligned}
$$

with $p_{\kappa+m} \in \Pi_{\kappa+m}(\zeta)$. We assume $\zeta^{(0)}(t)=1$ and $\zeta^{(j)}(t)=0$ for $j<0$ in (4.14).

(2) When $\kappa+m+1<0$, if and only if

$$
\frac{1}{2 \pi i} \int_{L_{0}} \frac{g(t)}{X^{+}(t)}\left[\zeta\left(t-G_{0}\right)-\zeta(t)\right] \mathrm{d} t=0
$$

and

$$
\frac{1}{2 \pi i} \int_{L_{0}} \frac{g(t)}{X^{+}(t)} \zeta^{(k)}(t) \mathrm{d} t=0, \quad k=0,1,2, \ldots,-\kappa-m-2,
$$

are satisfied, $D R_{m}$ problem (4.1) is solvable and its solution can be written as (4.15). We assume $\zeta^{(0)}(t)=1$ in (4.17).

In general, the freedom of solutions is $\kappa+m$. 


\section{Riemann BVP for doubly-periodic bianalytic functions}

In this section, we consider the following Riemann BVP for doubly-periodic bianalytic functions with the same factor: find a function $V \in D P H_{2}\left(S^{+} \cup S^{-} ; 2 \omega_{1}, 2 \omega_{2}\right)$ satisfying two Riemann-type boundary conditions and a growth condition

$$
\left\{\begin{array}{l}
V^{+}(t)=G(t) V^{-}(t)+g_{1}(t), \quad t \in L, \\
\left(\partial_{\bar{z}} V\right)^{+}(t)=G(t)\left(\partial_{\bar{z}} V\right)^{-}(t)+g_{2}(t), \quad t \in L, \\
\operatorname{Ord}(V, 0) \leq m,
\end{array}\right.
$$

where the given boundary datum $G$ and $g_{j}, j=1,2$, are Hölder-continuous on every curve $L_{k, \ell}$ and $G(t) \neq 0, t \in L$. In addition, $G\left(t+2 \omega_{j}\right)=G(t), g_{1}\left(t+2 \omega_{j}\right)=g(t), g_{2}\left(t+2 \omega_{j}\right)=g(t)$ for $j=1,2$ and $t \in L$. This problem is simply called $D B R_{m}$ problem.

Since $V \in \mathrm{DPH}_{2}\left(S^{+} \cup S^{-} ; 2 \omega_{1}, 2 \omega_{2}\right)$, by Theorem 2.1 or (2.12), one has the decomposition

$$
V(z)=V_{1}(z)+[\bar{z}-\lambda z-\delta \zeta(z)] V_{2}(z), \quad z \in S^{+} \cup S^{-},
$$

where $V_{j} \in D P H_{1}\left(\Omega ; 2 \omega_{1}, 2 \omega_{2}\right)$ for $j=1,2$.

Now, we will prove that $D B R_{m}$ problem (5.1) can be transformed to two independent $D R_{m+2}$ problem (5.3) and $D R_{m+1}$ problem (5.4) as follows:

$$
\left\{\begin{array}{l}
V_{1}^{+}(t)=G(t) V_{1}^{-}(t)+\tilde{g}_{1}(t), \quad t \in L, \\
\operatorname{Ord}\left(V_{1}, 0\right) \leq m+2
\end{array}\right.
$$

and

$$
\left\{\begin{array}{l}
V_{2}^{+}(t)=G(t) V_{2}^{-}(t)+g_{2}(t), \quad t \in L, \\
\operatorname{Ord}\left(V_{2}, 0\right) \leq m+1,
\end{array}\right.
$$

where

$$
\tilde{g}_{1}(t)=g_{1}(t)-[\bar{t}-\lambda t-\delta \zeta(t)] g_{2}(t)
$$

The solutions and conditions of solvability for those two problems have been presented in the preceding section.

Lemma 5.1 Let $V, V_{1}, V_{2}$ be given in (5.2). Then $V$ is the solution of $D B R_{m}$ problem (5.1) if and only if $V_{1}, V_{2}$ are respectively the solutions of $D R_{m+2}$ problem (5.3) and $D R_{m+1}$ problem (5.4) satisfying the relation

$$
\mathcal{L}_{j+1}\left[V_{1}\right]=\delta \mathcal{L}_{j}\left[V_{2}\right], \quad j=m, m+1,
$$

where $\mathcal{L}_{j}$ is the operator defined by (3.9), and $\delta$ is given in (2.4).

Proof Suppose that $V_{1}, V_{2}$ are respectively the solutions of $D R_{m+2}$ problem (5.3) and $D R_{m+1}$ problem (5.4) satisfying relation (5.6). By Theorem 3.1 and (5.6), $\operatorname{Ord}\left(V_{1}, 0\right) \leq m+2$ 
and $\operatorname{Ord}\left(V_{2}, 0\right) \leq m+1$ lead to

$$
\operatorname{Ord}(V, 0) \leq m
$$

On the other hand, one has

$$
\begin{aligned}
V^{+}(t) & =V_{1}^{+}(t)+\phi(t) V_{2}^{+}(t) \\
& =G(t)\left[V_{1}^{-}(t)+\phi(t) V_{2}^{-}(t)\right]+g_{1}(t)=G(t) V^{-}(t)+g_{1}(t),
\end{aligned}
$$

and

$$
\begin{aligned}
\left(\partial_{\bar{z}} V\right)^{+}(t) & =\left[\partial_{\bar{z}}\left(V_{1}+\phi V_{2}\right)\right]^{+}(t)=V_{2}^{+}(t)=G(t) V_{2}^{-}(t) \\
& =\left[\partial_{\bar{z}}\left(V_{1}+\phi V_{2}\right)\right]^{-}(t)=G(t)\left(\partial_{\bar{z}} V\right)^{-}(t)+g_{2}(t) .
\end{aligned}
$$

Combining (5.7), (5.8) with (5.9), $V$ is just a solution of $D B R_{m}$ problem (5.1).

Conversely, if $V$ is the solution of $D B R_{m}$ problem (5.1), obviously boundary conditions in (5.3) and (5.4) are valid. By Theorem 3.1, $\operatorname{Ord}(V, 0) \leq m$ implies $\operatorname{Ord}\left(V_{1}, 0\right) \leq m+2$ and $\operatorname{Ord}\left(V_{2}, 0\right) \leq m+1$, and the validity of relation (5.6). This completes the proof.

Analogously to the preceding section, we will discuss $D B R_{m}$ problem (5.1) in two cases according to $G_{*}=0\left(\bmod 2 \omega_{1}, 2 \omega_{2}\right)$ or $G_{*} \neq 0\left(\bmod 2 \omega_{1}, 2 \omega_{2}\right)$.

\subsection{The case $G_{*}=2 k \omega_{1}+2 \ell \omega_{2}$ for some $k, \ell \in \mathbb{Z}$}

In this case, $G_{*}=0\left(\bmod 2 \omega_{1}, 2 \omega_{2}\right)$. And we will discuss $D B R_{m}$ problem (5.1) in three subcases.

Theorem 5.1 If $\kappa+m+1>0, D B R_{m}$ problem (5.1) is solvable and its solution can be expressed as

$$
V(z)=X(z) W\left[g_{1}, g_{2}\right](z)+X(z)\left[p_{\kappa+m+1}(z)+\phi(z) q_{\kappa+m}(z)\right]
$$

with

$$
\mathcal{L}_{j+1}\left[p_{j+1}\right]=\delta \mathcal{L}_{j}\left[q_{j}\right], \quad j=\kappa+m, \kappa+m+1,
$$

where $p_{\kappa+m+1} \in \Pi_{\kappa+m+1}(\zeta), q_{\kappa+m} \in \Pi_{\kappa+m}(\zeta)$ and

$$
\begin{aligned}
W\left[g_{1}, g_{2}\right](z)= & \frac{1}{2 \pi i} \int_{L_{0}} \frac{g_{1}(t)}{X^{+}(t)}[\zeta(t-z)+\zeta(z)] \mathrm{d} t \\
& +\frac{1}{2 \pi i} \int_{L_{0}} \frac{g_{2}(t)}{X^{+}(t)}[\phi(z)-\phi(t)][\zeta(t-z)+\zeta(z)] \mathrm{d} t .
\end{aligned}
$$

Proof By Theorem 4.1, the solution of $D R_{m+2}$ problem (5.3) can be expressed as

$$
\begin{aligned}
V_{1}(z)= & \frac{X(z)}{2 \pi i} \int_{L_{0}} \frac{\widetilde{g}_{1}(t)}{X^{+}(t)}[\zeta(t-z)+\zeta(z)] \mathrm{d} t \\
& +X(z) p_{\kappa+m+1}(z), \quad p_{\kappa+m+1} \in \Pi_{\kappa+m+1}(\zeta),
\end{aligned}
$$


where $\Pi_{\kappa+m+1}(\zeta)$ is defined by (4.9) and $X$ is given by (4.6). By Theorem 4.1, the solution of $D R_{m+1}$ problem (5.4) can be expressed as

$$
V_{2}(z)=\frac{X(z)}{2 \pi i} \int_{L_{0}} \frac{g_{2}(t)}{X^{+}(t)}[\zeta(t-z)+\zeta(z)] \mathrm{d} t+X(z) p_{\kappa+m}(z), \quad p_{\kappa+m} \in \Pi_{\kappa+m}(\zeta) .
$$

According to Lemma 5.1, inserting (5.13) and (5.14) into (5.2), one gets

$$
\begin{aligned}
V(z)= & \frac{X(z)}{2 \pi i} \int_{L_{0}} \frac{g_{1}(t)-\phi(t) g_{2}(t)}{X^{+}(t)}[\zeta(t-z)+\zeta(z)] \mathrm{d} t+X(z) p_{\kappa+m+1}(z) \\
& +\frac{\phi(z) X(z)}{2 \pi i} \int_{L_{0}} \frac{g_{2}(t)}{X^{+}(t)}[\zeta(t-z)+\zeta(z)] \mathrm{d} t+\phi(z) X(z) p_{\kappa+m}(z) \\
= & \frac{X(z)}{2 \pi i} \int_{L_{0}} \frac{g_{1}(t)+[\phi(z)-\phi(t)] g_{2}(t)}{X^{+}(t)}[\zeta(t-z)+\zeta(z)] \mathrm{d} t \\
& +X(z)\left[p_{\kappa+m+1}(z)+\phi(z) p_{\kappa+m}(z)\right],
\end{aligned}
$$

which leads to (5.10). At the same time, (5.6) is reduced to (5.11). This completes the proof.

Remark 5.1 Under this case, combining (5.10) with (5.11), the solution of $D B R_{m}$ problem (5.1) can be rewritten as

$$
\begin{aligned}
V(z)= & X(z) W\left[g_{1}, g_{2}\right](z)+X(z)\left\{p_{\kappa+m-1}(z)+c_{\kappa+m} \zeta^{(\kappa+m)}(z)+c_{\kappa+m+1} \zeta^{(\kappa+m+1)}(z)\right. \\
& \left.+\phi(z)\left[q_{\kappa+m-2}(z)+\frac{c_{\kappa+m}}{\delta} \zeta^{(\kappa+m-1)}(z)+\frac{c_{\kappa+m+1}}{\delta} \zeta^{(\kappa+m)}(z)\right]\right\},
\end{aligned}
$$

where $p_{\kappa+m-1} \in \Pi_{\kappa+m-1}(\zeta), q_{\kappa+m-2} \in \Pi_{\kappa+m-2}(\zeta), c_{\kappa+m} \in \mathbb{C}$ and $c_{\kappa+m+1} \in \mathbb{C}$.

Theorem 5.2 If $\kappa+m+1=0$, if and only if

$$
\frac{1}{2 \pi i} \int_{L_{0}} \frac{g_{2}(t)}{X^{+}(t)} \mathrm{d} t=0
$$

$D B R_{m}$ problem (5.1) is solvable and its solution can be represented as

$$
V(z)=X(z) \widetilde{W}\left[g_{1}, g_{2}\right](z)+\frac{C}{\delta} X(z)[\delta+\phi(z)], \quad C \in \mathbb{C},
$$

with

$$
\mathcal{L}_{-1}\left[\left\{\widetilde{W}\left[g_{1}, g_{2}\right]\right\}_{0}\right]=\delta \mathcal{L}_{-2}\left[\left\{\widetilde{W}\left[g_{1}, g_{2}\right]\right\}_{1}\right],
$$

where $\left\{\widetilde{W}\left[g_{1}, g_{2}\right]\right\}_{j}$ is j-component of $\widetilde{W}\left[g_{1}, g_{2}\right]$ defined by

$$
\widetilde{W}\left[g_{1}, g_{2}\right](z)=W\left[g_{1}, g_{2}\right](z)-\frac{\phi(z)}{2 \pi i} \int_{L_{0}} \frac{g_{2}(t)}{X^{+}(t)}[\zeta(z)+\zeta(t)] \mathrm{d} t
$$


Proof By Theorem 4.1, the solution of $D R_{m+2}$ problem (5.3) can be expressed as

$$
V_{1}(z)=\frac{X(z)}{2 \pi i} \int_{L_{0}} \frac{\widetilde{g}_{1}(t)}{X^{+}(t)}[\zeta(t-z)+\zeta(z)] \mathrm{d} t+X(z) C_{1}, \quad C_{1} \in \mathbb{C}
$$

By Theorem 4.1, if and only if the condition of solvability (5.15) is fulfilled, the solution of $D R_{m+1}$ problem (5.4) can be expressed as

$$
V_{2}(z)=\frac{X(z)}{2 \pi i} \int_{L_{0}} \frac{g_{2}(t)}{X^{+}(t)}[\zeta(t-z)-\zeta(t)] \mathrm{d} t+X(z) C_{2}, \quad C_{2} \in \mathbb{C}
$$

By Lemma 5.1, putting (5.19) and (5.20) into (5.2), one easily gets

$$
\begin{aligned}
V(z)= & X(z)\left\{\frac{1}{2 \pi i} \int_{L_{0}} \frac{g_{1}(t)-\phi(t) g_{2}(t)}{X^{+}(t)}[\zeta(t-z)+\zeta(z)] \mathrm{d} t\right. \\
& \left.+\frac{\phi(z)}{2 \pi i} \int_{L_{0}} \frac{g_{2}(t)}{X^{+}(t)}[\zeta(t-z)-\zeta(t)] \mathrm{d} t\right\} \\
& +X(z)\left[C_{1}+\phi(z) C_{2}\right],
\end{aligned}
$$

with $C_{1}, C_{2} \in \mathbb{C}$. Also by Lemma $5.1, V$ given by (5.21) is the solution of $D B R_{m}$ problem (5.1) if and only if $C_{1}=\delta C_{2}$ and (5.17) are satisfied. And hence the proof of the theorem is completed.

Theorem 5.3 If $\kappa+m+1<0$, if and only if

$$
\frac{1}{2 \pi i} \int_{L_{0}} \frac{g_{1}(t)-\phi(t) g_{2}(t)}{X^{+}(t)} \zeta^{(k)}(t) \mathrm{d} t=0, \quad k=-1,0,1,2, \ldots,-\kappa-m-3
$$

and

$$
\frac{1}{2 \pi i} \int_{L_{0}} \frac{g_{2}(t)}{X^{+}(t)} \zeta^{(k)}(t) \mathrm{d} t=0, \quad k=-1,0,1,2, \ldots,-\kappa-m-2,
$$

$D B R_{m}$ problem (5.1) is solvable and its solution can be represented as

$$
V(z)=X(z)\left\{\widehat{W}\left[g_{1}, g_{2}\right](z)+C\right\}
$$

with

$$
\left\{\begin{array}{l}
C=\delta \mathcal{L}_{\kappa+m+1}\left[\left\{\widehat{W}\left[g_{1}, g_{2}\right]\right\}_{1}\right] \\
\mathcal{L}_{\kappa+m+1}\left[\left\{\widehat{W}\left[g_{1}, g_{2}\right]\right\}_{0}\right]=\delta \mathcal{L}_{\kappa+m}\left[\left\{\widehat{W}\left[g_{1}, g_{2}\right]\right\}_{1}\right]
\end{array}\right.
$$

where $\left\{\widehat{W}\left[g_{1}, g_{2}\right]\right\}_{j}$ is j-component of $\widehat{W}\left[g_{1}, g_{2}\right]$ defined by

$$
\begin{aligned}
\widehat{W}\left[g_{1}, g_{2}\right](z)= & \frac{1}{2 \pi i} \int_{L_{0}} \frac{g_{1}(t)}{X^{+}(t)}[\zeta(t-z)-\zeta(t)] \mathrm{d} t \\
& +\frac{1}{2 \pi i} \int_{L_{0}} \frac{g_{2}(t)}{X^{+}(t)}[\phi(z)-\phi(t)][\zeta(t-z)-\zeta(t)] \mathrm{d} t .
\end{aligned}
$$


Proof By Theorem 4.1, if and only if the conditions of solvability (5.22) are fulfilled, the solution of $D R_{m+2}$ problem (5.3) can be expressed as

$$
\begin{aligned}
V_{1}(z)= & \frac{X(z)}{2 \pi i} \int_{L_{0}} \frac{g_{1}(t)-\phi(t) g_{2}(t)}{X^{+}(t)}[\zeta(t-z)-\zeta(t)] \mathrm{d} t \\
& +C X(z), \quad C \in \Pi_{\kappa+m+2}(\zeta) .
\end{aligned}
$$

Analogously, by Theorem 4.1, if and only if the conditions of solvability (5.23) are satisfied, the solution of $D R_{m+2}$ problem (5.4) can be written as

$$
V_{2}(z)=\frac{X(z)}{2 \pi i} \int_{L_{0}} \frac{g_{2}(t)}{X^{+}(t)}[\zeta(t-z)-\zeta(t)] \mathrm{d} t
$$

Therefore, by Lemma 5.1, if and only if the conditions of solvability (5.22) and (5.23) are fulfilled, the solution of $D B R_{m}$ problem (5.1) can be expressed as

$$
\begin{aligned}
V(z)= & \frac{X(z)}{2 \pi i} \int_{L_{0}} \frac{g_{1}(t)-\phi(t) g_{2}(t)}{X^{+}(t)}[\zeta(t-z)-\zeta(t)] \mathrm{d} t+X(z) C \\
& +\frac{\phi(z) X(z)}{2 \pi i} \int_{L_{0}} \frac{g_{2}(t)}{X^{+}(t)}[\zeta(t-z)-\zeta(t)] \mathrm{d} t \\
= & \frac{X(z)}{2 \pi i} \int_{L_{0}} \frac{g_{1}(t)+[\phi(z)-\phi(t)] g_{2}(t)}{X^{+}(t)}[\zeta(t-z)-\zeta(t)] \mathrm{d} t \\
& +X(z) C, \quad C \in \Pi_{\kappa+m+2}(\zeta),
\end{aligned}
$$

satisfying relation (5.25). This completes the proof.

\subsection{The case $G_{*} \neq 2 k \omega_{1}+2 \ell \omega_{2}$ for any $k, \ell \in \mathbb{Z}$}

In this case, there exists $G_{0} \in S_{0}$ such that $G_{*}=G_{0}\left(\bmod 2 \omega_{1}, 2 \omega_{2}\right)$, and $G_{0} \neq 0$. We will investigate the problem in four subcases.

Theorem 5.4 If $\kappa+m+2 \geq 0, D B R_{m}$ problem (5.1) is solvable and its solution can be expressed as

$$
\begin{aligned}
V(z)= & X(z)\left\{W\left[g_{1}, g_{2}\right](z)-W\left[g_{1}, g_{2}\right]\left(G_{0}\right)\right\} \\
& +X(z)\left\{\left[p_{\kappa+m+2}(z)-p_{\kappa+m+2}\left(G_{0}\right)\right]+\phi(z)\left[q_{\kappa+m+1}(z)-q_{\kappa+m+1}\left(G_{0}\right)\right]\right\},
\end{aligned}
$$

with

$$
\mathcal{L}_{j+1}\left[p_{j+1}\right]=\delta \mathcal{L}_{j}\left[q_{j}\right], \quad j=\kappa+m+1, \kappa+m+2,
$$

and $p_{\kappa+m+1} \in \Pi_{\kappa+m+1}(\zeta), q_{\kappa+m} \in \Pi_{\kappa+m}(\zeta)$, where $X$ is the same as that in Sect. 4.2 and $W\left[g_{1}, g_{2}\right](z)$ is given in (5.12).

Proof By Theorem 4.2, the solution of $D R_{m+2}$ problem (5.3) can be expressed as

$$
\begin{aligned}
V_{1}(z)= & \frac{X(z)}{2 \pi i} \int_{L_{0}} \frac{g_{1}(t)-\phi(t) g_{2}(t)}{X^{+}(t)}\left[\zeta(t-z)+\zeta(z)-\zeta\left(t-G_{0}\right)-\zeta\left(G_{0}\right)\right] \mathrm{d} t \\
& +X(z)\left[p_{\kappa+m+2}(z)-p_{\kappa+m+2}\left(G_{0}\right)\right]
\end{aligned}
$$


with $p_{\kappa+m+2} \in \Pi_{\kappa+m+2}(\zeta)$, where $\Pi_{\kappa+m+2}(\zeta)$ is defined by (4.9) and $X$ is given by (4.6) with (4.13). By Theorem 4.2, the solution of $D R_{m+1}$ problem (5.4) can be represented as

$$
\begin{aligned}
V_{2}(z)= & \frac{X(z)}{2 \pi i} \int_{L_{0}} \frac{g_{2}(t)}{X^{+}(t)}\left[\zeta(t-z)+\zeta(z)-\zeta\left(t-G_{0}\right)-\zeta\left(G_{0}\right)\right] \mathrm{d} t \\
& +X(z)\left[p_{\kappa+m+1}(z)-p_{\kappa+m+1}\left(G_{0}\right)\right],
\end{aligned}
$$

with $p_{\kappa+m+1} \in \Pi_{\kappa+m+1}(\zeta)$. Observe

$$
\begin{aligned}
& W\left[g_{1}, g_{2}\right](z)-W\left[g_{1}, g_{2}\right]\left(G_{0}\right) \\
& =\frac{1}{2 \pi i} \int_{L_{0}} \frac{g_{1}(t)-\phi(t) g_{2}(t)}{X^{+}(t)}\left[\zeta(t-z)+\zeta(z)-\zeta\left(t-G_{0}\right)-\zeta\left(G_{0}\right)\right] \mathrm{d} t \\
& \quad+\frac{\phi(z)}{2 \pi i} \int_{L_{0}} \frac{g_{2}(t)}{X^{+}(t)}\left[\zeta(t-z)+\zeta(z)-\zeta\left(t-G_{0}\right)-\zeta\left(G_{0}\right)\right] .
\end{aligned}
$$

Inserting (5.31) and (5.32) into (5.2), one easily gets expression (5.29). By Lemma 5.1, (5.29) is the solution of $D B R_{m}$ problem (5.1) if and only if (5.30) is satisfied.

Theorem 5.5 If $\kappa+m+2=-1$, if and only if

$$
\frac{1}{2 \pi i} \int_{L_{0}} \frac{g_{2}(t)}{X^{+}(t)} \mathrm{d} t=0
$$

$D B R_{m}$ problem (5.1) is solvable and its solution can be expressed as

$$
V(z)=X(z)\left\{W\left[g_{1}, g_{2}\right](z)-W\left[g_{1}, g_{2}\right]\left(G_{0}\right)\right\},
$$

with

$$
\mathcal{L}_{j+1}\left[\left\{W\left[g_{1}, g_{2}\right]\right\}_{0}\right]=\delta \mathcal{L}_{j}\left[\left\{W\left[g_{1}, g_{2}\right]\right\}_{1}\right], \quad j=-1,0,
$$

where $W\left[g_{1}, g_{2}\right](z)$ is given by (5.12).

Proof By Theorem 4.2, the solution of $D R_{m+2}$ problem (5.3) can be expressed as

$$
V_{1}(z)=\frac{X(z)}{2 \pi i} \int_{L_{0}} \frac{g_{1}(t)-\phi(t) g_{2}(t)}{X^{+}(t)}\left[\zeta(t-z)+\zeta(z)-\zeta\left(t-G_{0}\right)-\zeta\left(G_{0}\right)\right] \mathrm{d} t .
$$

By Theorem 4.2, if and only if the condition of solvability (5.33) is fulfilled, the solution of $D R_{m+1}$ problem (5.4) can be written as

$$
V_{2}(z)=\frac{X(z)}{2 \pi i} \int_{L_{0}} \frac{g_{2}(t)}{X^{+}(t)}\left[\zeta(t-z)-\zeta\left(t-G_{0}\right)\right] \mathrm{d} t .
$$

By (5.33), expression (5.37) can be rewritten as

$$
V_{2}(z)=\frac{X(z)}{2 \pi i} \int_{L_{0}} \frac{g_{2}(t)}{X^{+}(t)}\left[\zeta(t-z)+\zeta(z)-\zeta\left(t-G_{0}\right)-\zeta\left(G_{0}\right)\right] \mathrm{d} t .
$$


Thus, by Lemma 5.1, similar to the preceding discussion, the desired conclusion is obtained.

Theorem 5.6 If $\kappa+m+2<-1$, if and only if

$$
\begin{aligned}
& \frac{1}{2 \pi i} \int_{L_{0}} \frac{g_{1}(t)-\phi(t) g_{2}(t)}{X^{+}(t)}\left[\zeta\left(t-G_{0}\right)-\zeta(t)\right] \mathrm{d} t=0, \\
& \frac{1}{2 \pi i} \int_{L_{0}} \frac{g_{2}(t)}{X^{+}(t)}\left[\zeta\left(t-G_{0}\right)-\zeta(t)\right] \mathrm{d} t=0, \\
& \frac{1}{2 \pi i} \int_{L_{0}} \frac{g_{1}(t)-\phi(t) g_{2}(t)}{X^{+}(t)} \zeta^{(k)}(t) \mathrm{d} t=0, \quad k=0,1,2, \ldots,-\kappa-m-4
\end{aligned}
$$

and

$$
\frac{1}{2 \pi i} \int_{L_{0}} \frac{g_{2}(t)}{X^{+}(t)} \zeta^{(k)}(t) \mathrm{d} t=0, \quad k=0,1,2, \ldots,-\kappa-m-3
$$

$D B R_{m}$ problem (5.1) is solvable and its solution can be expressed as

$$
V(z)=X(z)\left\{W\left[g_{1}, g_{2}\right](z)-W\left[g_{1}, g_{2}\right]\left(G_{0}\right)\right\},
$$

with

$$
\mathcal{L}_{j+1}\left[\left\{W\left[g_{1}, g_{2}\right]\right\}_{0}\right]=\delta \mathcal{L}_{j}\left[\left\{W\left[g_{1}, g_{2}\right]\right\}_{1}\right], \quad j=\kappa+m+1, \kappa+m+2,
$$

where $W\left[g_{1}, g_{2}\right](z)$ is given by (5.12).

Proof By Theorem 4.2, if and only if conditions (5.38) and (5.40) are fulfilled, the solution of $D R_{m+2}$ problem (5.3) can be expressed as

$$
V_{1}(z)=\frac{X(z)}{2 \pi i} \int_{L_{0}} \frac{g_{1}(t)-\phi(t) g_{2}(t)}{X^{+}(t)}\left[\zeta(t-z)-\zeta\left(t-G_{0}\right)\right] \mathrm{d} t .
$$

By Theorem 4.2, if and only if the conditions of solvability (5.39) and (5.41) are fulfilled, the solution of $D R_{m+1}$ problem (5.4) can be written as

$$
V_{2}(z)=\frac{X(z)}{2 \pi i} \int_{L_{0}} \frac{g_{2}(t)}{X^{+}(t)}\left[\zeta(t-z)-\zeta\left(t-G_{0}\right)\right] \mathrm{d} t
$$

And hence, analogously to the preceding discussion, the desired conclusion is obtained.

Remark 5.2 To sum up the discussion above, the freedom of solutions of $D B R_{m}$ problem $(5.1)$ is $2(\kappa+m)+1$.

\section{Conclusion}

In this article, we define doubly-periodic polyanalytic functions and growth order of doubly-periodic polyanalytic functions at the origin. Riemann BVP of doubly-periodic bianalytic functions is presented. The problem is transformed into two independent 
Riemann-type BVPs of doubly-periodic analytic functions. Finally, the solution is explicitly expressed as the integral representation.

Boundary value problems are always related with the theory of elasticity (see, for example, $[24,27,28])$. If the stresses and the elastic region are doubly periodic, BVPs of doubly-periodic functions can be applied to the theory of planar elasticity. Furthermore, the number and the shape of cracks in the so-called fundamental periodic parallelogram described in Sect. 2 could be arbitrary. In some sense, the results obtained here could contribute to the investigation of planar elasticity of doubly-periodic functions.

Acknowledgements

The authors would like to thank the referees for their valuable suggestions which helped to improve this work.

\section{Funding}

This research is supported by Major Innovation Projects for Building First-class Universities in China's Western Region (ZKZD2017009).

\section{Availability of data and materials}

Not applicable.

\section{Competing interests}

The authors declare that they have no competing interests.

\section{Consent for publication}

Not applicable.

\section{Authors' contributions}

$\mathrm{HH}$ carried out theoretical calculation, participated in the design of the study, and drafted the manuscript. HL conceived of the study and participated in the design of the study. YW participated in its design and helped to draft the manuscript. All authors read and approved the final manuscript.

\section{Author details}

${ }^{1}$ School of Mathematics and Statistics, Ningxia University, Yinchuan, P.R. China. ${ }^{2}$ Department of Mathematics, Tianjin University of Technology and Education, Tianjin, P.R. China. ${ }^{3}$ School of Mathematics and Statistics, Wuhan University, Wuhan, P.R. China.

\section{Publisher's Note}

Springer Nature remains neutral with regard to jurisdictional claims in published maps and institutional affiliations.

Received: 26 November 2017 Accepted: 23 May 2018 Published online: 31 May 2018

\section{References}

1. Balk, M.B.: Polyanalytic Functions. Akademie Verlag, Berlin (2001)

2. Gonchar, A.A., Havin, V.P., Nikolski, N.K. (eds.): Complex Analysis I: Entire and Meromorphic Functions, Polyanalytic Functions and Their Generalizations. Springer, Berlin (1997)

3. Begehr, H., Schmersau, D.: The Schwarz problem for polyanalytic functions. Z. Anal. Anwend. 24(2), 341-351 (2005)

4. Begehr, H., Hile, G.N.: A hierarchy of integral operators. Rocky Mt. J. Math. 27, 669-706 (1997)

5. Begehr, H., Kumar, A.: Boundary value problem for inhomogeneous polyanalytic equation I. Analysis 25, 55-71 (2005)

6. Begehr, H., Vanegas, C.J.: Iterated Neumann problem for higher order Poisson equation. Math. Nachr. 279, 38-57 (2006)

7. Begehr, H., Du, J.Y., Wang, Y.F.: A Dirichlet problem for polyharmonic functions. Ann. Mat. Pura Appl. 187(3), 435-457 (2008)

8. Du, Z.H., Qian, T., Wang, J.X.: $L_{p}$ polyharmonic Dirichlet problems in regular domains II: the upper half plane. J. Differ. Equ. 252, 1789-1812 (2012)

9. Fatulaev, B.F: The main Haseman type boundary value problem for metaanalytic function in the case of circular domains. Math. Model. Anal. 6(1), 68-76 (2001)

10. Wang, Y.F., Wang, Y.J.: On Riemann problems for single-periodic polyanalytic functions. Math. Nachr. 287(16), 1886-1915 (2014)

11. Han, P.J., Wang, Y.F.: A note on Riemann problems for single-periodic polyanalytic functions. Math. Nachr. 289(13), 1594-1605 (2016)

12. Wang, Y.F., Du, J.Y.: On Riemann boundary value problem of polyanalytic functions on the real axis. Acta Math. Sci. 24B(4), 663-671 (2004)

13. Du, J.Y., Wang, Y.F: Riemann boundary value problems of polyanalytic functions and metaanalytic functions on the closed curves. Complex Var. Theory Appl. 50(7-11), 521-533 (2005)

14. Du, J.Y., Wang, Y.F: On boundary value problems of polyanalytic function on the real axis. Complex Var. Theory Appl. 48(6), 527-542 (2003) 
15. Wang, Y.F.: On modified Hilbert boundary-value problems of polyanalytic functions. Math. Methods Appl. Sci. 32, 1415-1427 (2009)

16. Wang, Y.F: Schwarz-type boundary value problems of polyanalytic equation on the upper half unit disk. Complex Var. Elliptic Equ. 57(9), 983-993 (2012)

17. Wang, Y.F.: On Hilbert-type boundary-value problem of poly-Hardy class on the unit disc. Complex Var. Elliptic Equ. 58(4), 497-509 (2013)

18. Wang, Y.F., Wang, Y.J. Schwarz-type problem of nonhomogeneous Cauchy-Riemann equation on a triangle. J. Math. Anal. Appl. 377, 557-570 (2011)

19. Wang, Y., Wang, Y.F.: Two boundary-value problems for the Cauchy-Riemann equation in a sector. Complex Anal. Oper. Theory 6, 1121-1138 (2012)

20. Wang, Y.F., Han, P.J., Wang, Y.J.: On Riemann problem of automorphic polyanalytic functions connected with a rotation group. Complex Var. Elliptic Equ. 60(8), 1033-1057 (2015)

21. Begehr, H.: Complex Analytic Methods for Partial Differential Equation: An Introductory Text. World Scientific, Singapore (1994)

22. Kopperlman, K:: The Riemann-Hilbert problem for finite Riemann surfaces. Commun. Pure Appl. Math. 12, 13-35 (1959)

23. Rodin, YL:The Riemann Boundary Problem on Riemann Surfaces, Reidel, Dordrecht (1988)

24. Lu, J.K.: Boundary Value Problems for Analytic Functions. World Scientific, Singapore (1993)

25. Gakhov, F.D.: Boundary Value Problems. Pergamon, Oxford (1966)

26. Dang, P., Du, J.Y., Qian, T.: Boundary value problems for periodic analytic functions. Bound. Value Probl. 2015, 143 (2015)

27. Marin, M.: On weak solutions in elasticity of dipolar bodies with voids. J. Comput. Appl. Math. 82(1-2), 291-297 (1997)

28. Cai, H.T., Lu, J.K.: Mathematical Theory in Periodic Plane Elasticity. Gordon \& Breach, Singapore (2000)

\section{Submit your manuscript to a SpringerOpen ${ }^{\circ}$ journal and benefit from:}

- Convenient online submission

- Rigorous peer review

- Open access: articles freely available online

- High visibility within the field

- Retaining the copyright to your article

Submit your next manuscript at $\boldsymbol{\nabla}$ springeropen.com 